\title{
Remoção de nutrientes por Typha latifolia e Cynodon spp. cultivadas em sistemas alagados construídos
}

(doi:10.4136/ambi-agua.175)

\section{Fátima Resende Luiz Fia ${ }^{1}$; Antonio Teixeira de Matos ${ }^{2}$; Ronaldo Fia ${ }^{1}$; Túlio Ferreira Lambert $^{2}$; Mateus Pimentel de Matos ${ }^{2}$}

\author{
${ }^{1}$ Departamento de Engenharia - Universidade Federal de Lavras \\ E-mail: \{fatimarlf, ronaldofia\}@deg.ufla.br \\ ${ }^{2}$ Departamento de Engenharia Agrícola - Universidade Federal de Viçosa \\ E-mail: atmatos@ufv.br; tulioflambert@hotmail.com; mateus.matos@ufv.br
}

\section{RESUMO}

Objetivou-se, neste trabalho, avaliar a capacidade extratora de duas espécies vegetais quando cultivadas em sistemas alagados construídos de escoamento subsuperficial horizontal (SACs) para o tratamento das águas residuárias da suinocultura (ARS). Para tal, foram construídos 8 SACs de 2,0 m x 0,5 m x 0,6 m, em fibra de vidro, preenchidos com 0,55 m de brita zero. Nos $\mathrm{SAC}_{2} ; \mathrm{SAC}_{4} ; \mathrm{SAC}_{6}$ e $\mathrm{SAC}_{8}$, foi cultivada a taboa (Typha latifolia) e nos $\mathrm{SAC}_{3}$; $\mathrm{SAC}_{5}$; $\mathrm{SAC}_{7}$ e $\mathrm{SAC}_{9}$ foi cultivado o capim tifton-85 (Cynodon spp.). Os $\mathrm{SAC}_{2}$ e $\mathrm{SAC}_{3}$, $\mathrm{SAC}_{4}$ e $\mathrm{SAC}_{5}, \mathrm{SAC}_{6}$ e $\mathrm{SAC}_{7}$ e $\mathrm{SAC}_{8}$ e $\mathrm{SAC}_{9}$ receberam 163, 327, 461 e $561 \mathrm{~kg} \mathrm{ha}^{-1} \mathrm{~d}^{-1}$ de DBO, respectivamente. Durante os 120 dias de monitoramento dos SACs, verificou-se que a taboa não se adaptou às condições de exposição avaliadas. As maiores produtividades foram obtidas com a aplicação da carga orgânica média de $327 \mathrm{~kg} \mathrm{ha}^{-1} \mathrm{~d}^{-1}$ de DBO. O capim tifton85 foi a espécie vegetal que apresentou maior capacidade extratora de nutrientes, chegando a extrair entre 443 e 540, 86 e 99, 193 e 241, 0,77 e 2,17, e 1,21 e 3,68 kg ha ${ }^{-1}$ de NTK, P, K, $\mathrm{Cu}$ e $\mathrm{Zn}$, respectivamente, enquanto a taboa apresentou maior capacidade de absorção de sódio.

Palavras-chave: macrófita; nutrientes; extração; sistemas wetlands; água residuária da suinocultura.

\section{Nutrients removal by Typha latifolia and Cynodon spp. grown in constructed wetlands}

\begin{abstract}
The objective of this study was to evaluate the extraction capacity of two species when grown in constructed wetlands with subsurface horizontal flow (SACs) for the treatment of swine wastewater (ARS). To this end, were built 8 SACs of $2.0 \mathrm{~m} \times 0.5 \mathrm{~m} \times 0.6 \mathrm{~m}$, fiber glass, filled with $0.55 \mathrm{~m}$ of fine gravels. In $\mathrm{SAC}_{2} ; \mathrm{SAC}_{4} ; \mathrm{SAC}_{6}$ and $\mathrm{SAC}_{8}$ was cultivated cattail (Typha latifolia) and in $\mathrm{SAC}_{3} ; \mathrm{SAC}_{5} ; \mathrm{SAC}_{7}$ and $\mathrm{SAC}_{9}$ was cultivated tifton-85 bermudagrass (Cynodon spp.). The $\mathrm{SAC}_{2}$ and $\mathrm{SAC}_{3}, \mathrm{SAC}_{4}$ and $\mathrm{SAC}_{5}, \mathrm{SAC}_{6}$ and $\mathrm{SAC}_{7}$ and $\mathrm{SAC}_{8}$ and $\mathrm{SAC}_{9}$ received 163, 327, 461 and $561 \mathrm{~kg} \mathrm{ha}^{-1}$ day $^{-1}$ of BOD, respectively. During the 120 days of the SACs monitoring, it was found that the cattail has not adapted to the conditions of exposure. The highest yields were obtained with the application of organic load average of $327 \mathrm{~kg} \mathrm{ha}^{-1} \mathrm{day}^{-1}$ of BOD. The tifton-85 was the plant species with the highest capacity to extract nutrients, getting to draw between 443 and 540, 86 and 99, 193 and 241, 0.77 and 2.17, and 1.21 and $3.68 \mathrm{~kg} \mathrm{ha}^{-1} \mathrm{TKN}, \mathrm{P}, \mathrm{K}, \mathrm{Cu}$ and Zn, respectively, while cattail showed greater capacity to absorb sodium.
\end{abstract}

Keywords: macrophyte; nutrients; extraction; wetlands; swine wastewater. 


\section{INTRODUÇÃO}

Há uma grande variedade de macrófitas aquáticas que podem ser utilizadas no tratamento de águas residuárias em sistemas alagados construídos (SACs). Todavia, é necessário que essas plantas tenham tolerância à inundação contínua e exposição à água geralmente rica em sais, além de possuir crescimento rápido e alta capacidade de remoção de nutrientes (Brasil et al., 2007a). Em SACs pesquisados no Brasil, têm sido usadas as espécies: Typha spp. (Brasil et al., 2007a; Matos et al., 2009), Juncus spp. (Sousa et al., 2000), Eleocharis spp. (Mazzola et al., 2005), arroz (Oriza sativa L.) (Meira et al., 2004) e gramíneas do gênero Cynodon (Matos et al., 2008, 2009). Além de outras como alternantera (Alternanthera philoxeroides) (Matos et al., 2009; Fia et al., 2008), azevém (Lolium multiflorum) (Fia et al., 2010) e lírio amarelo (Hemerocallis flava) (Chagas, 2008).

As macrófitas apresentam várias propriedades intrínsecas que as tornam um componente indispensável nos leitos cultivados, sendo que as funções mais importantes das macrófitas consistem nos efeitos físicos proporcionados ao tratamento. A presença da vegetação em SACs distribui e reduz a velocidade do escoamento, o que cria melhores condições para sedimentação de sólidos suspensos, reduzindo o risco de erosão e ressuspensão de sólidos já sedimentados, e aumenta o tempo de contato entre a água e as raízes das plantas (Brix, 1997).

Por meio dos mecanismos de absorção e assimilação, as plantas são capazes de remover nutrientes das águas poluídas, muitos dos quais ficam presos no sistema pelos sucessivos ciclos de crescimento, morte e decomposição (Kadlec et al., 2005). Essas plantas removem, ainda, substâncias contendo metais pesados e compostos orgânicos tóxicos. No entanto, para sustentar a alta produtividade e retirar do sistema os nutrientes nele incorporados, é necessário, também, que haja o manejo adequado das plantas, com colheitas periódicas da biomassa (Kadlec e Wallace, 2008). Geralmente, SACs vegetados são mais eficientes na remoção de poluentes quando comparados aos sem vegetação, principalmente porque a rizosfera da planta estimula a densidade e atividade da comunidade microbiana, fornecendo à superfície da raiz para o crescimento microbiano e fontes de carbono por meio de exsudatos radiculares e por oferecer um microambiente aeróbico via liberação de oxigênio pela raiz (Brix, 1997; Bertin et al., 2003), além de absorver nutrientes solubilizados no meio. Contudo, a influência da vegetação na remoção dos compostos orgânicos em sistemas alagados construídos não é unânime, ainda assim, a maioria dos estudos indica o efeito positivo das macrófitas.

No presente trabalho, teve-se como objetivo estudar o comportamento agronômico e a extração de macronutrientes pela taboa (Typha latifolia) e pelo capim tifton-85 (Cynodon spp.) no tratamento das águas residuárias da suinocultura (ARS), em sistemas alagados construídos (SACs), quando submetidos a diferentes cargas orgânicas.

\section{MATERIAL E MÉTODOS}

O experimento foi constituído por oito sistemas alagados de escoamento subsuperficial horizontal (SACs), implantados em casa de vegetação, constituídos por caixas de fibra de vidro, nas dimensões de 0,6 m de altura x $0,5 \mathrm{~m}$ de largura x 2,0 m de comprimento, assentadas sobre o solo, em declividade de $0,01 \mathrm{~m} \mathrm{~m}^{-1}$; preenchidos com brita zero (volume de vazios inicial de $0,494 \mathrm{~m}^{3} \mathrm{~m}^{-3}$ ) até a altura de $0,55 \mathrm{~m}$, deixando-se uma borda livre de 0,05 $\mathrm{m}$. O nível d'água foi mantido a $0,05 \mathrm{~m}$ abaixo da superfície do material suporte.

Nos $\mathrm{SAC}_{2}, \mathrm{SAC}_{4}, \mathrm{SAC}_{6}$ e $\mathrm{SAC}_{8}$ foi plantada a taboa (Typha latifolia) e nos $\mathrm{SAC}_{3}, \mathrm{SAC}_{5}$, $\mathrm{SAC}_{7}$ e $\mathrm{SAC}_{9}$ foi plantado o capim tifton-85 (Cynodon spp.). No plantio da taboa foram utilizados 14 propágulos vegetativos (rizomas) por $\mathrm{m}^{2}$ e 20 segmentos de caule do capim tifton-85, com quatro a seis nós, para cada $\mathrm{m}^{2}$. 
Após um mês de implantação do sistema, iniciou-se a aplicação da água residuária da suinocultura (ARS) aos SACs. A ARS utilizada no experimento foi proveniente de um tanque de alvenaria com 8,5 m de largura, $14 \mathrm{~m}$ de comprimento e profundidade média de 1,8 m, que recebia as águas da lavagem das baias do Setor de Suinocultura do Departamento de Zootecnia da UFV. Nesse tanque, a ARS passava por um tratamento anaeróbio, devido às características deste e ao volume de efluente gerado. As variações nas principais características da ARS ao longo de todo período de experimentação estão apresentadas na Tabela 1. Para caracterização da ARS foi utilizada a metodologia descrita por APHA et al. (2005).

Tabela 1. Valores médios e desvio-padrão das principais características da ARS utilizada durante o monitoramento do sistema.

\begin{tabular}{l|r}
\hline Variáveis & \multicolumn{1}{|c}{ ARS } \\
\hline $\mathrm{pH}$ & $7,6 \pm 0,3^{(12)}$ \\
$\left.\mathrm{CE}(\mathrm{dS} \mathrm{m})^{-1}\right)$ & $4,30 \pm 0,41^{(12)}$ \\
$\mathrm{DQO}\left(\mathrm{mg} \mathrm{L}^{-1}\right)$ & $2.492 \pm 1.705^{(12)}$ \\
$\mathrm{DBO}\left(\mathrm{mg} \mathrm{L}^{-1}\right)$ & $777 \pm 519^{(12)}$ \\
$\mathrm{SST}\left(\mathrm{mg} \mathrm{L}^{-1}\right)$ & $953 \pm 803^{(12)}$ \\
$\mathrm{NTK}\left(\mathrm{mg} \mathrm{L}^{-1}\right)$ & $530 \pm 96^{(12)}$ \\
$\mathrm{N}-\mathrm{NH}_{4}^{+}\left(\mathrm{mg} \mathrm{L}^{-1}\right)$ & $401 \pm 121^{(11)}$ \\
$\mathrm{P}\left(\mathrm{mg} \mathrm{L}^{-1}\right)$ & $161 \pm 119^{(12)}$ \\
$\mathrm{K}\left(\mathrm{mg} \mathrm{L}^{-1}\right)$ & $103 \pm 70^{(10)}$ \\
$\mathrm{Na}\left(\mathrm{mg} \mathrm{L}^{-1}\right)$ & $79 \pm 55^{(10)}$ \\
$\mathrm{Ca}\left(\mathrm{mg} \mathrm{L}^{-1}\right)$ & $38 \pm 14^{(7)}$ \\
$\mathrm{Mg}\left(\mathrm{mg} \mathrm{L}^{-1}\right)$ & $17 \pm 6^{(7)}$ \\
$\mathrm{Cu}\left(\mathrm{mg} \mathrm{L}^{-1}\right)$ & $0,09 \pm 0,07^{(7)}$ \\
$\mathrm{Zn}\left(\mathrm{mg} \mathrm{L}^{-1}\right)$ & $0,75 \pm 0,56^{(7)}$ \\
\hline
\end{tabular}

(a) Entre parênteses o número de amostragens consideradas no cálculo das médias.

A aplicação da carga orgânica foi feita de forma diferenciada nos SACs, por meio da variação na vazão afluente aos sistemas, cujas características operacionais estão apresentadas na Tabela 2. A alimentação dos SACs foi feita por meio de bomba dosadora a solenóide, da marca ProMinent ${ }^{\circledR}$ CONCEPT. Para obtenção das variáveis operacionais dos SACs, considerou-se o volume útil deles, obtido pelo produto do volume total de cada SAC pela porosidade do material suporte.

Aos 60 e aos 120 dias após o início da aplicação da ARS e monitoramento dos sistemas, foram realizados os cortes dos vegetais, a fim de se quantificar a produtividade e os teores de nutrientes na parte aérea da vegetação e, com isso, calcular a capacidade das plantas cultivadas nos SACs em extrair nutrientes/poluentes da ARS neles aplicada.

As amostras coletadas dos tecidos vegetais foram pesadas e levadas à estufa, sob temperatura de $65^{\circ} \mathrm{C}$, com circulação de ar, até ser atingida massa constante. Em seguida, as amostras foram trituradas em moinho para realização da análise nutricional. Parte da amostra de cada SAC foi colocada em estufa, sob temperatura de $103-105^{\circ} \mathrm{C}$, até ser atingida constância na massa, obtendo-se a matéria seca total, em cada tratamento. Além da matéria seca, nos vegetais, foram quantificados os teores de macronutrientes (NTK, P e K) e sódio, seguindo-se metodologia proposta pela EMBRAPA (1999). 
Tabela 2. Características operacionais médias e desvio padrão dos oito SACs.

\begin{tabular}{l|cc}
\hline Variáveis & SAC $_{\mathbf{2}}$ & SAC $_{3}$ \\
\hline $\mathrm{Q}$ & $0,021 \pm 0,002 \mathrm{a}$ & $0,021 \pm 0,002 \mathrm{a}$ \\
$\mathrm{TDH}$ & $11,8 \pm 1,1 \mathrm{a}$ & $12,0 \pm 1,2 \mathrm{a}$ \\
$\mathrm{TCO}_{\mathrm{A}}$ & $164 \pm 110 \mathrm{a}$ & $162 \pm 108 \mathrm{a}$ \\
\hline & $\mathrm{SAC}_{4}$ & SAC $_{5}$ \\
\hline $\mathrm{Q}$ & $0,042 \pm 0,004 \mathrm{~b}$ & $0,042 \pm 0,005 \mathrm{~b}$ \\
$\mathrm{TDH}$ & $5,9 \pm 0,6 \mathrm{~b}$ & $5,9 \pm 0,6 \mathrm{~b}$ \\
$\mathrm{TCO}_{\mathrm{A}}$ & $327 \pm 219 \mathrm{~b}$ & $327 \pm 219 \mathrm{~b}$ \\
\hline & $\mathrm{SAC}_{\mathbf{6}}$ & $\mathbf{S A C}_{\mathbf{7}}$ \\
\hline $\mathrm{Q}$ & $0,059 \pm 0,005 \mathrm{c}$ & $0,059 \pm 0,004 \mathrm{c}$ \\
$\mathrm{TDH}$ & $4,2 \pm 0,4 \mathrm{c}$ & $4,2 \pm 0,3 \mathrm{c}$ \\
$\mathrm{TCO}_{\mathrm{A}}$ & $460 \pm 308 \mathrm{c}$ & $461 \pm 308 \mathrm{c}$ \\
\hline & $\mathrm{SAC}_{\mathbf{8}}$ & $\mathrm{SAC}_{\mathbf{9}}$ \\
\hline $\mathrm{Q}$ & $0,066 \pm 0,017 \mathrm{~d}$ & $0,078 \pm 0,006 \mathrm{e}$ \\
$\mathrm{TDH}$ & $4,0 \pm 1,1 \mathrm{~d}$ & $3,2 \pm 0,3 \mathrm{e}$ \\
$\mathrm{TCO}_{\mathrm{A}}$ & $515 \pm 344 \mathrm{~d}$ & $607 \pm 406 \mathrm{~d}$ \\
\hline
\end{tabular}

$\mathrm{Q}$ - vazão afluente $\left(\mathrm{m}^{3} \mathrm{~d}^{-1}\right)$, TDH - tempo de detenção hidráulica (d), $\mathrm{TCO}_{\mathrm{A}}$ - Taxa de carga orgânica tomada com base na área superficial $\left(\mathrm{kg} \mathrm{ha}^{-1} \mathrm{~d}^{-1} \mathrm{de} \mathrm{DBO}\right)$. Médias das mesmas variáveis seguidas de mesma letra não diferem significativamente entre si, a 5\% de probabilidade, pelo teste de Tukey.

\section{RESULTADOS E DISCUSSÃO}

\subsection{Desempenho das espécies cultivadas nos SACs: produção de matéria seca}

Nenhuma das espécies avaliadas (Typha latifolia e Cynodon spp.) apresentou problemas de adaptação no meio, antes dos SACs serem submetidos à aplicação da ARS. Entretanto, já aos 15 dias após o início da aplicação da ARS, observou-se o amarelecimento do tecido foliar da taboa, nos SACs em que foram aplicadas as maiores cargas orgânicas $\left(\mathrm{SAC}_{6} \mathrm{e} \mathrm{SAC}_{8}\right)$. Aos 30 dias de monitoramento do sistema, verificou-se senescência das folhas da taboa nos $\mathrm{SAC}_{6}$ e $\mathrm{SAC}_{8}$ e os primeiros sinais de senescência foliar nos $\mathrm{SAC}_{2}$ e $\mathrm{SAC}_{4}$, também cultivados com a taboa. Quanto ao capim tifton-85, não se verificou sinais de senescência (Figura 1A).

Após o primeiro corte dos vegetais houve rebrotamento em todos os SACs. No entanto, a taboa cultivada nos $\mathrm{SAC}_{4}, \mathrm{SAC}_{6}$ e $\mathrm{SAC}_{8}$ voltou a apresentar sinais de senescência, enquanto as plantas cultivadas no $\mathrm{SAC}_{2}$ apresentaram reduzido crescimento vegetativo, indicando baixa capacidade de adaptação dessa espécie vegetal às condições do meio (Figura 1B). 
A.
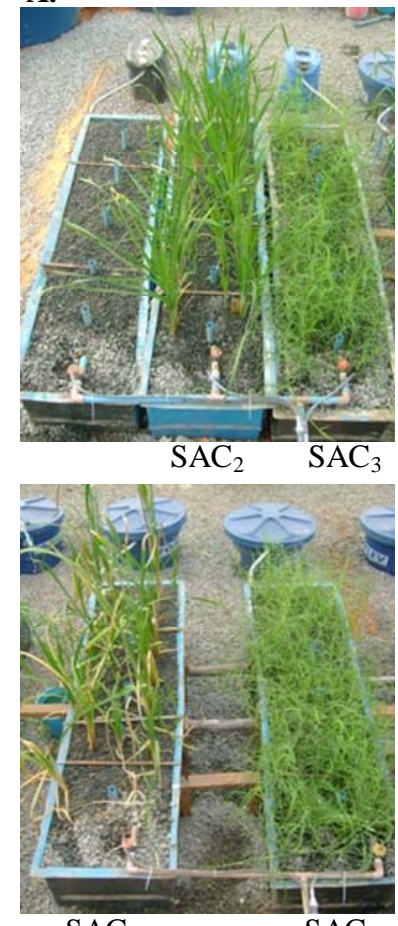

$\mathrm{SAC}_{6}$
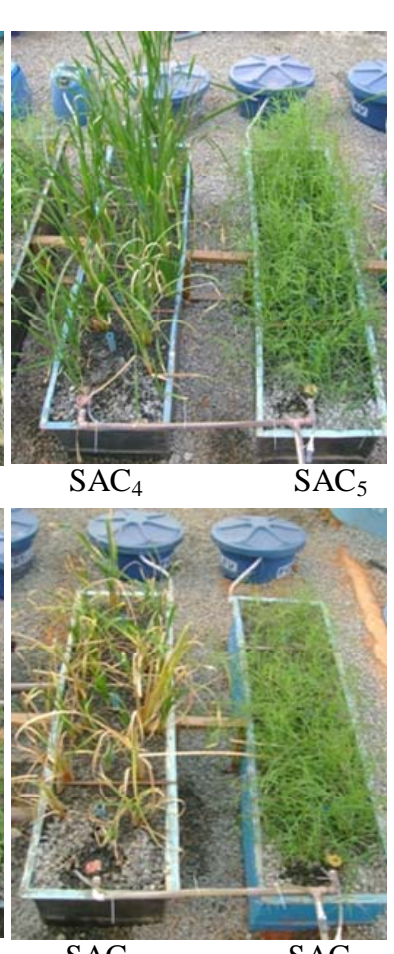

B.
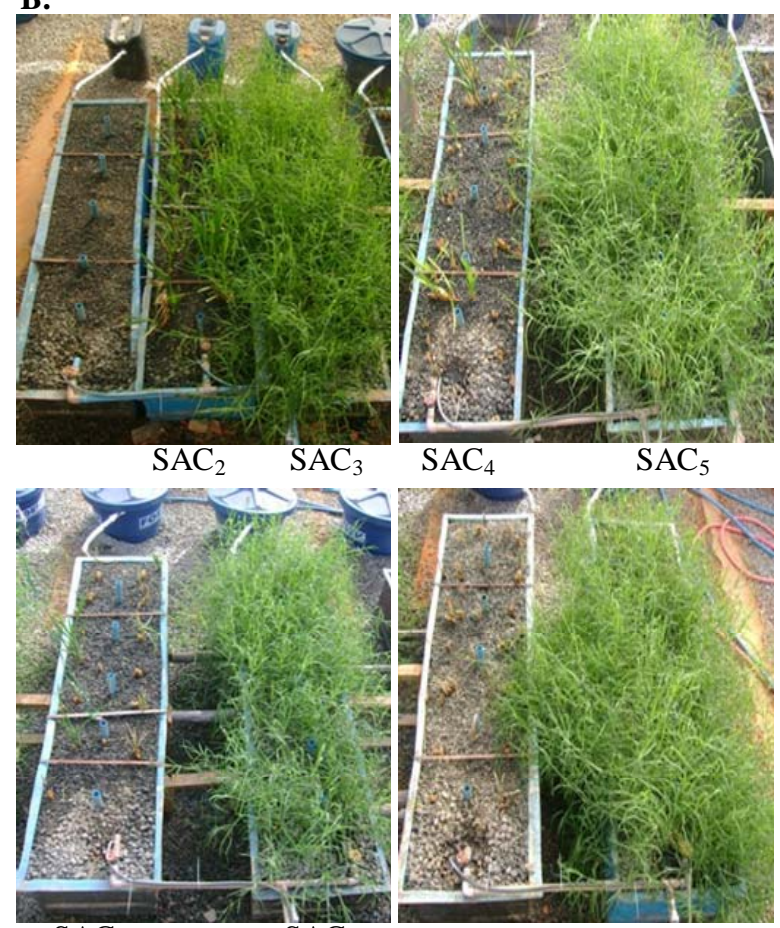

$\mathrm{SAC}_{6}$

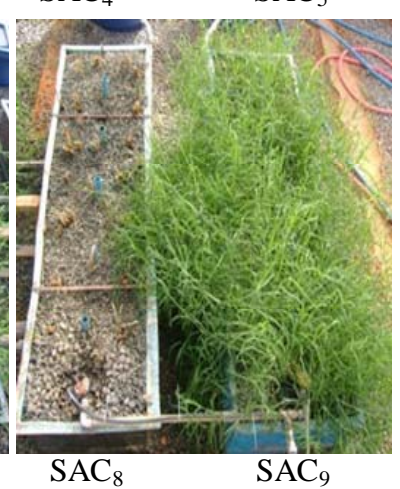

Figura 1. (A) Primeiros sinais de senescência apresentados pela taboa após 15 dias do início da aplicação contínua da ARS; (B) Rebrotamento das espécies vegetais após o primeiro corte realizado aos 60 dias após o início de operação do sistema.

Na Figura 2, está apresentada a produtividade de matéria seca obtida nos cortes 1 e 2, evidenciando o efeito da carga orgânica aplicada aos SACs no valor dessa variável.

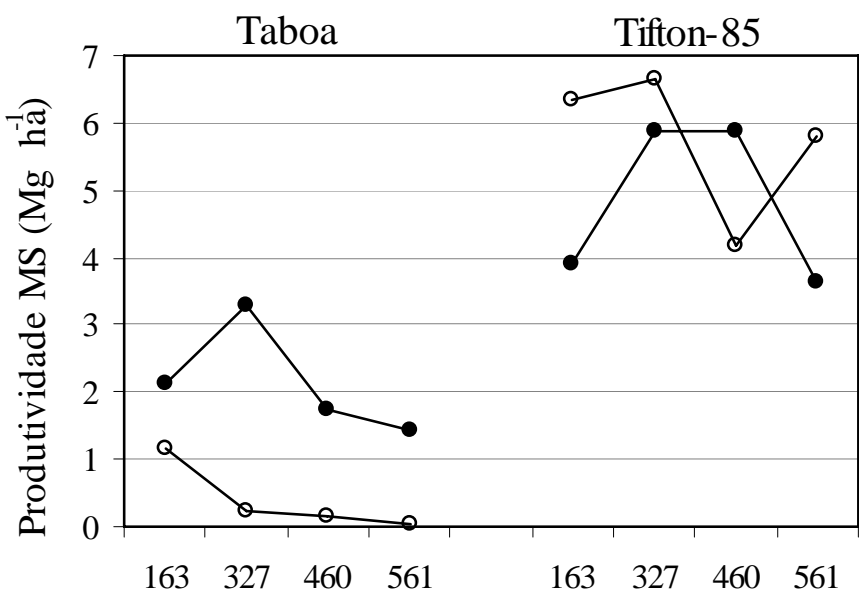

Cargas médias $\left(\mathrm{kg} \mathrm{ha}^{-1} \mathrm{~d}^{-1}\right.$ de DBO)

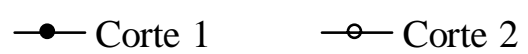

Figura 2. Valores médios da produtividade de matéria seca obtidos nos cortes 1 e 2, evidenciando o efeito da carga orgânica aplicada sobre a produção de matéria seca de capim tifton-85 e taboa.

Deve-se ressaltar que a matéria seca avaliada no primeiro corte foi produzida durante um período de 90 dias, sendo 30 dias de adaptação e 60 dias de aplicação das cargas orgânicas diferenciadas de ARS. A matéria seca quantificada no segundo corte foi produzida em um período de 60 dias. 
Verificou-se que a produtividade de matéria seca pelo capim tifton-85 no segundo corte foi, na maioria dos SACs, cultivados com esse vegetal (exceção o $\mathrm{SAC}_{7}$ ), superior às obtidas no primeiro corte. Após o primeiro corte, observou-se que, no $\mathrm{SAC}_{7}$, apareceram falhas de plantas (espaços vazios), o que foi causado pelo mau desenvolvimento das mudas plantadas no início do experimento, já que antes do primeiro corte, apresentava-se totalmente coberto pela vegetação, devido ao intenso perfilhamento do capim tifton-85. Matos et al. (2009) não verificaram problemas de adaptação do capim tifton-85 aos SACs, no entanto foi constatada dificuldade em se atingir a densidade de plantas adequadas para cultivo nos sistemas alagados utilizados no tratamento de efluentes da suinocultura.

Em relação à taboa, observou-se redução na quantidade de matéria seca produzida, em todos os SACs, no segundo corte. Em relação às cargas aplicadas aos SACs, tanto de matéria orgânica como de nutrientes, notou-se que a taboa não se adaptou ao ambiente de cultivo. Acredita-se que a dificuldade da taboa em desenvolver seus propágulos nos SACs não tenha sido devido, somente, às cargas orgânicas aplicadas, visto que outros autores trabalharam com cargas orgânicas superiores e obtiveram bom desenvolvimento dela, mas em função das concentrações elevadas de nutrientes, os quais podem aumentar a salinidade e, consequentemente, reduzir o potencial osmótico do meio, além da toxicidade causada pelo excesso de nutrientes.

Brasil et al. (2007b) constataram que a taboa, aos 100 dias de cultivo, apresentou sinais de fitotoxicidade e senescência, ao ser cultivada em sistemas utilizados no tratamento da ARS (concentrações de N, P, K e Na de 820 a 3.092; 240 a 1.700; 680 a 2.540 e 743 a $2.107 \mathrm{mg} \mathrm{L}^{-1}$, respectivamente). Os autores associaram os danos causados à taboa ao efeito de salinidade da ARS. Clarke e Baldwin (2002) afirmaram que concentrações de amônio superiores a $200 \mathrm{mg} \mathrm{L}^{-1}$ reduziram a taxa de crescimento da taboa, valor este inferior ao das concentrações médias observadas na ARS (Tabela 1), o que também pode ter sido fator de impedimento ao desenvolvimento das plantas. Acredita-se que um conjunto de fatores associados à maior concentração de K e Na na ARS utilizada neste trabalho, associados à intoxicação com sódio tenham afetado o desenvolvimento satisfatório da taboa, principalmente nos SACs que receberam as maiores cargas orgânicas.

Quanto à taboa, houve tendência de redução na produtividade de matéria seca com o aumento da carga orgânica aplicada, enquanto que no caso do capim tifton-85 as cargas orgânicas intermediárias mostraram-se mais adequadas ao seu desenvolvimento vegetativo. No entanto, para as duas espécies vegetais, não houve diferença estatística $(\mathrm{P}<0,05)$ entre as taxas de carga orgânica aplicada (tratamentos). Matos et al. (2008) verificaram aumento na produtividade de matéria seca do capim tifton-85 cultivado em SACs receptores de maiores taxas de carga orgânica provenientes das águas residuárias de laticínios. Entretanto, valores superiores a $500 \mathrm{~kg} \mathrm{ha}^{-1} \mathrm{~d}^{-1}$ de DBO provocaram tendência de redução na produtividade de matéria seca, tendo sido a máxima produtividade de, aproximadamente, $15 \mathrm{Mg} \mathrm{ha}^{-1}$, obtida aos 45 dias de cultivo do capim tifton-85. Matos et al. (2009) obtiveram produtividades médias de matéria seca de 22 e $28 \mathrm{Mg} \mathrm{ha}^{-1}$ de taboa e capim tifton 85 , respectivamente, quando cultivados por períodos entre 60 e 90 dias em SACs utilizados no tratamento de águas residuárias da suinocultura. Queiroz et al. (2004) obtiveram 17,7 $\mathrm{Mg}$ ha $^{-1}$ de matéria seca de capim tifton-85 quando fertirrigado com águas residuárias da suinocultura aplicadas numa taxa de $800 \mathrm{~kg} \mathrm{ha}^{-1} \mathrm{~d}^{-1}$ de DBO. Essa produtividade é superior à obtida em sistemas convencionais de cultivo no solo, em que são utilizados adubos químicos e irrigação que, segundo Oliveira et al. (2000), deve estar entre 3 e $12 \mathrm{Mg} \mathrm{ha}^{-1}$, aos 14 e 70 dias de cultivo, após o corte. 
FIA, F. R. L.; MATOS, A. T.; FIA, R.; LAMBERT, T. F.; MATOS, M. P. Remoção de nutrientes por Typha latifolia e Cynodon spp. cultivadas em sistemas alagados construídos. Ambi-Agua, Taubaté, v. 6, n. 1, p. 77-89, 2011. (doi:10.4136/ambi-agua.175)

\subsection{Desempenho das espécies cultivadas nos SACs: remoção de macronutrientes e sódio}

Os dados médios da avaliação dos macronutrientes e sódio contidos na parte aérea das espécies avaliadas, obtidos nos dois cortes realizados durante a condução do experimento, estão apresentados na Tabela 3.

Tabela 3. Concentrações médias e desvio-padrão de macronutrientes e sódio na matéria seca foliar da taboa e do capim tifton-85, obtidas nos dois cortes realizados durante a condução do experimento.

\begin{tabular}{|c|c|c|c|c|c|c|c|c|}
\hline \multirow{3}{*}{ Nutrientes } & \multicolumn{4}{|c|}{ Taboa } & \multicolumn{4}{|c|}{ Capim tifton-85 } \\
\hline & $\mathrm{SAC}_{2}$ & $\mathrm{SAC}_{4}$ & $\mathrm{SAC}_{6}$ & $\mathrm{SAC}_{8}$ & $\mathrm{SAC}_{3}$ & $\mathrm{SAC}_{5}$ & $\mathrm{SAC}_{7}$ & $\mathrm{SAC}_{9}$ \\
\hline & \multicolumn{8}{|c|}{------------------------------------- dag kg } \\
\hline NTK & 3,11 & 2,99 & 3,12 & 3,27 & 4,55 & 4,31 & 4,47 & 4,68 \\
\hline PB & - & - & - & - & 28,4 & 27,0 & 28,0 & 29,2 \\
\hline $\mathrm{P}$ & 0,20 & 0,25 & 0,25 & 0,32 & 0,95 & 0,79 & 0,97 & 0,91 \\
\hline $\mathrm{K}$ & 2,29 & 2,57 & 2,61 & 3,38 & 2,11 & 1,93 & 2,10 & 2,04 \\
\hline $\mathrm{Na}$ & 0,97 & 0,81 & 0,75 & 0,92 & 0,02 & 0,03 & 0,02 & 0,02 \\
\hline
\end{tabular}

PB - Proteína bruta obtida pela multiplicação do teor de nitrogênio por 6,25. $\mathrm{SAC}_{2}$ e $\mathrm{SAC}_{3}, \mathrm{SAC}_{4}$ e $\mathrm{SAC}_{5}, \mathrm{SAC}_{6}$ e $\mathrm{SAC}_{7}$ e $\mathrm{SAC}_{8}$ e $\mathrm{SAC}_{9}$ receberam, respectivamente, 163, 327, 460 e $561 \mathrm{~kg} \mathrm{ha}^{-1} \mathrm{~d}^{-1}$ de DBO.

Verifica-se que foram encontradas maiores concentrações de nitrogênio e de fósforo na matéria seca do tecido foliar do capim tifton-85 quando comparado à da taboa, ao se avaliar as mesmas cargas orgânicas aplicadas aos SACs. O inverso foi observado em relação ao potássio e o sódio.

Segundo a FAO (2006), as concentrações típicas encontradas no tecido foliar da taboa variam de 0,2 a 2,$4 ; 0,14$ a 0,$20 ; 0,88$ a 2,65 e 0,18 a 0,28 dag kg-1 $^{-1}$, para $\mathrm{N}, \mathrm{P}, \mathrm{K}$ e $\mathrm{Na}$. Queiroz (2000) e Matos et al. (2008) obtiveram menores valores de PB no capim tifton-85, quando cultivado sob a aplicação de várias cargas orgânicas de diferentes águas residuárias. Queiroz (2000), ao fertirrigar o capim tifton-85 com água residuária da suinocultura, obteve 19 dag $\mathrm{kg}^{-1}$ de PB no referido capim. Matos et al. (2008), ao utilizarem SACs cultivados com capim tifton-85 no tratamento de águas residuárias de laticínios, obtiveram teores de PB entre 18 e 25 dag $\mathrm{kg}^{-1}$. Oliveira et al. (2000) constataram que os valores de PB no capim tifton-85 cultivado convencionalmente, em diferentes idades, pode variar de 4,5 a 15,6 dag $\mathrm{kg}^{-1}$. Tal como o ocorrido em relação à proteína bruta, que é calculada a partir da concentração de nitrogênio na matéria seca, os valores de nitrogênio encontrados na matéria seca do capim tifton-85, obtidos neste trabalho, foram superiores aos encontrados pelos autores citados anteriormente. De forma semelhante, os valores de nitrogênio obtidos na matéria seca da taboa também foram superiores aos relatados pela FAO (2006).

A concentração de fósforo na matéria seca foliar da taboa variou com os tratamentos aplicados, sendo os valores encontrados semelhantes aos reportados por Brasil et al. (2007a) e inferiores aos obtidos por Matos et al. (2009), que foram, respectivamente, de 0,28 e 0,44 dag $\mathrm{kg}^{-1}$. Quanto ao capim tifton-85, as concentrações de fósforo na matéria seca foram superiores às observadas por Oliveira et al. (2000) que, ao cultivarem o capim tifton-85 sob condições de pastagem convencional, obtiveram concentrações foliares entre 0,16 e 0,27 dag kg-1 . Mesmo trabalhando com águas residuárias, sabidamente ricas em nutrientes, Matos et al. (2008), Loures et al. (2006) e Queiroz et al. (2004) obtiveram concentrações foliares de fósforo, em capim tifton-85, próximas à metade daquelas encontradas neste trabalho (Tabela 3), tendo esses autores obtido, respectivamente, concentrações entre 0,32 e 0,41 dag $\mathrm{kg}^{-1} ; 0,46$ dag $\mathrm{kg}^{-1}$; e 0,34 e 0,37 dag $\mathrm{kg}^{-1}$.

Fia et al. (2008) verificaram que a concentração de potássio no tecido foliar da taboa aumentou, variando entre 2 e 7 dag $\mathrm{kg}^{-1}$, com o aumento na carga orgânica aplicada, ao trabalhar com SACs no tratamento das águas do processamento dos frutos do cafeeiro, sabidamente ricas em potássio. Matos et al. (2009) e Brasil et al. (2007a) encontraram, 
respectivamente, valores de 2,42 e 2,83 dag $\mathrm{kg}^{-1}$ de $\mathrm{K}$, valores esses mais próximos aos encontrados neste trabalho. Matos et al. (2008) obtiveram concentrações de potássio inferiores a 1,5 dag $\mathrm{kg}^{-1}$ no tecido vegetal do capim tifton-85, em todos os tratamentos aplicados. Oliveira et al. (2000) obtiveram, no mesmo capim, aos 70 dias de cultivo em solo com a adição de adubos químicos, 1,28 dag $\mathrm{kg}^{-1}$ de $\mathrm{K}$. Diante dos resultados, pode-se considerar que os valores obtidos neste experimento encontram-se na faixa considerada adequada para as plantas e são semelhantes à maior parte dos reportados na literatura.

Brasil et al. (2007a) e Matos et al. (2009) obtiveram 0,80 e 0,46 dag $\mathrm{kg}^{-1}$ de sódio nas folhas da taboa ao tratarem esgoto doméstico e águas residuárias da suinocultura, respectivamente, em SACs, sendo esses valores inferiores aos obtidos neste trabalho. Acredita-se que isso tenha ocorrido devido às maiores cargas de sódio aplicadas neste trabalho, quando comparadas às aplicadas nos outros dois. Além disso, a taboa parece ter capacidade de absorver mais sódio, quando há disponibilidade desse nutriente, quando comparado ao capim tifton-85. Apesar de Silva (2008) ter obtido concentrações de sódio na matéria seca do capim tifton-85 da ordem de $0,25 \mathrm{dag} \mathrm{kg}^{-1}$, os valores obtidos neste trabalho foram semelhantes aos obtidos por esse mesmo autor no cultivo do referido capim com água de mananciais superficiais e adubação química. Valores semelhantes aos obtidos neste trabalho também foram encontrados por Matos et al. (2008) e Queiroz et al. (2004) que, ao trabalharem com águas residuárias de laticínios e da suinocultura, obtiveram, respectivamente, 0,03 e 0,04 dag kg-1 de sódio nas folhas do capim tifton-85.

Na Figura 3 estão apresentados os valores médios de remoção de macronutrientes e sódio em dois cortes da parte aérea dos vegetais, em função da carga de nutrientes aplicadas aos SACs, durante os 120 dias de monitoramento.

O capim tifton-85 destacou-se em relação à taboa na remoção de nutrientes, exceto no que se refere ao sódio. Apesar de a taboa ter apresentado maior concentração de nutrientes e sódio no tecido foliar (exceto nitrogênio e fósforo), em razão do menor efeito diluição, a produtividade de biomassa foi o fator responsável pelas maiores porcentagens de remoção apresentadas pelo capim tifton-85. Os dados apresentados na Figura 3 evidenciam a capacidade das plantas em remover nutrientes e sódio e que essa remoção torna-se mais evidente quando são aplicadas as menores cargas de nutrientes nos SACs, apesar de não ter sido verificada, neste trabalho, diferença estatística $(p<0,05)$ entre os tratamentos.

As extrações de nitrogênio obtidas, neste trabalho, pelo capim tifton-85 foram próximas aos $552 \mathrm{~kg} \mathrm{ha}^{-1}$ obtidos por Queiroz (2000) em experimento conduzido em solo fertirrigado com ARS, com carga aplicada de $175 \mathrm{~kg} \mathrm{ha}^{-1} \mathrm{~d}^{-1}$ de $\mathrm{N}$, em quatro meses de monitoramento do sistema. No entanto, as extrações de fósforo foram superiores aos $61 \mathrm{~kg} \mathrm{ha}^{-1}$, obtidas pelo mesmo autor, ao aplicar uma carga de fósforo superior às aplicadas neste trabalho ( $\left.91 \mathrm{~kg} \mathrm{ha}^{-1} \mathrm{~d}^{-1}\right)$. Matos et al. (2009) obtiveram remoções médias, durante 110 dias de monitoramento, de 681 e $107 \mathrm{~kg} \mathrm{ha}^{-1}$ de $\mathrm{N}$ e P, por meio de cortes efetuados em capim tifton-85 cultivado em SACs utilizados no tratamento de ARS. Com o corte da taboa, os mesmos autores obtiveram 533 e $88 \mathrm{~kg} \mathrm{ha}^{-1}$ de N e P, resultados semelhantes aos obtidos neste trabalho. Matos et al. (2009) concluíram que o capim tifton-85 foi muito mais eficiente na remoção de nitrogênio e fósforo que a taboa.

As extrações de $\mathrm{K}$ e Na obtidas neste trabalho também foram inferiores às obtidas por Queiroz et al. (2004), que obteve extração de $591 \mathrm{~kg} \mathrm{ha}^{-1}$ de $\mathrm{K} \mathrm{e} \mathrm{6,6} \mathrm{kg}^{-1} \mathrm{ha}^{-1}$ de $\mathrm{Na}$ em solo fertirrigado com ARS, aplicada numa carga de 91 e $15 \mathrm{~kg} \mathrm{ha}^{-1} \mathrm{~d}^{-1}$ de $\mathrm{K}$ e $\mathrm{Na}$, respectivamente, em 4 meses de aplicação. 


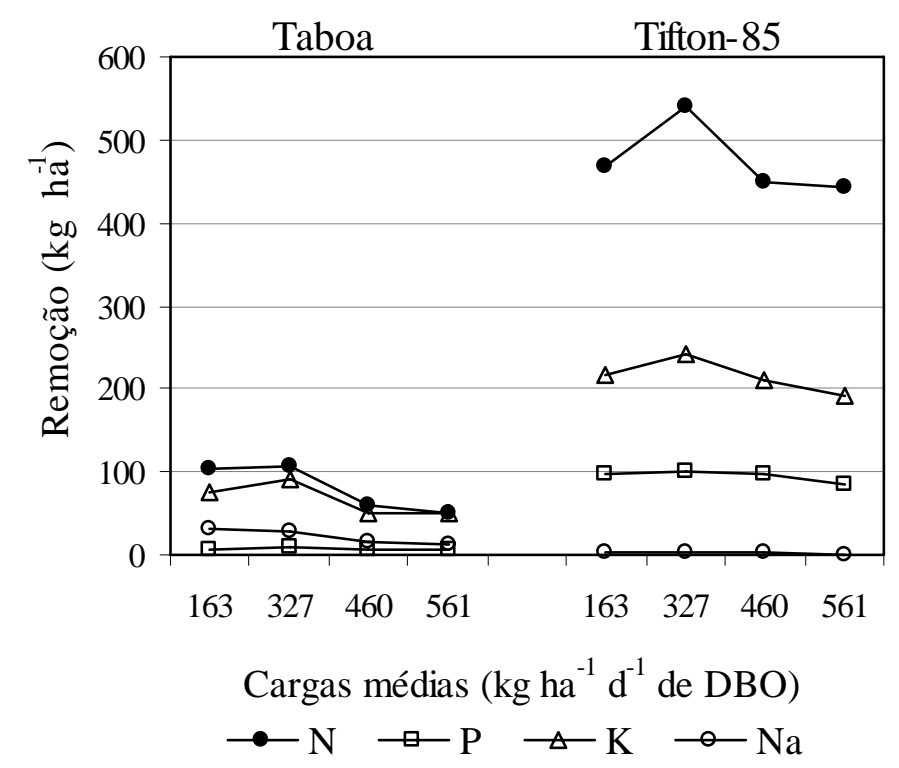

Figura 3. Valores médios de remoção $\left(\mathrm{kg} \mathrm{ha}^{-1}\right)$ de nitrogênio (NTK), fósforo (P), potássio (K) e sódio $(\mathrm{Na})$, obtidos em dois cortes nas plantas, realizados em 120 dias de operação dos SACs, em função das cargas orgânicas aplicadas.

Semelhante ao que foi observado neste trabalho, os dados apresentados por Matos et al. (2009) evidenciam reduzida capacidade de extração de sódio pelo capim tifton-85, quando comparado com a taboa. A menor capacidade de absorção desse elemento químico também foi observada por Matos et al. (2008) e Queiroz et al. (2004). Esse fato pode ter favorecido o estabelecimento e manutenção do capim tifton-85 nos SACs, uma vez que a taboa, por ser uma planta acumuladora de $\mathrm{Na}$, apresentou sinais de intoxicação que podem estar associados à presença, em excesso, desse cátion em seus tecidos.

\subsection{Desempenho das espécies cultivadas nos SACs: remoção de micronutrientes}

Os dados médios da avaliação de concentração dos micronutrientes $\mathrm{Cu}$ e $\mathrm{Zn}$ na parte aérea das espécies avaliadas, obtidos nos dois cortes realizados durante a condução do experimento, estão apresentados na Tabela 4.

Tabela 4. Concentrações médias de cobre (Cu) e zinco ( $\mathrm{Zn}$ ) na matéria seca foliar da taboa e do capim tifton-85, obtidas nos dois cortes realizados da parte aérea dos vegetais, durante a condução do experimento.

\begin{tabular}{|c|c|c|c|c|c|c|c|c|}
\hline \multirow{3}{*}{ Nutrientes } & \multicolumn{4}{|c|}{ Taboa } & \multicolumn{4}{|c|}{ Capim tifton-85 } \\
\hline & $\mathrm{SAC}_{2}$ & $\mathrm{SAC}_{4}$ & $\mathrm{SAC}_{6}$ & $\mathrm{SAC}_{8}$ & $\mathrm{SAC}_{3}$ & $\mathrm{SAC}_{5}$ & $\mathrm{SAC}_{7}$ & $\mathrm{SAC}_{9}$ \\
\hline & \multicolumn{8}{|c|}{ 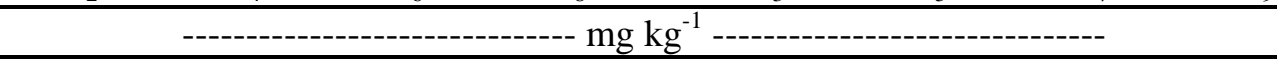 } \\
\hline $\mathrm{Cu}$ & 46,9 & 31,3 & 62,5 & 28,1 & 75,0 & 172,9 & 39,6 & 164,6 \\
\hline $\mathrm{Zn}$ & 707,5 & 250,0 & 382,5 & 516,9 & 210,6 & 294,2 & 120,2 & 314,2 \\
\hline
\end{tabular}

Verifica-se que, na matéria seca do tecido foliar do capim tifton-85, foram encontrados maiores concentrações de cobre, quando comparados aos resultados obtidos quando foram aplicadas as mesmas cargas orgânicas aos SACs cultivados com taboa, sendo o inverso observado em relação ao zinco. Observa-se na Tabela 4 que não houve tendência de aumento nas concentrações de Zn tanto na taboa como no capim tifton-85 e de Cu no capim tifton-85 com o aumento das cargas orgânicas aplicadas. A concentração foliar de $\mathrm{Cu}$ na taboa manteve-se relativamente constante com aumento da carga orgânica aplicada. 
Sasmaz et al. (2008), ao avaliarem plantas de taboa que se desenvolveram em sistemas alagados naturais que recebiam esgotos domésticos, na Turquia, obtiveram concentrações foliares de 30 e $215 \mathrm{mg} \mathrm{kg}^{-1}$ de $\mathrm{Cu}$ e $\mathrm{Zn}$. As concentrações de $\mathrm{Cu}$ encontradas na parte aérea das plantas, neste trabalho, ficaram próximas, porém ainda superiores, enquanto as de $\mathrm{Zn}$ foram de uma a três vezes superiores às obtidas na Turquia. Os autores relataram, ainda, que o $\mathrm{Cu}$ e o $\mathrm{Zn}$, apesar de serem micronutrientes essenciais às plantas, podem ser extremamente tóxicos a elas, quando presente em concentrações relativamente altas. Normalmente as concentrações de Zn não excedem $100 \mathrm{mg} \mathrm{kg}^{-1}$ em plantas cultivadas em ambientes naturais não poluídos. Os mesmos autores concluíram que a taboa não foi eficiente na translocação de metais das raízes para a parte aérea, o que reduziu sua capacidade de extrair metais de ambientes poluídos, no entanto possui grande tolerância a elevadas concentrações de Cu e Zn no meio de seu cultivo.

Na Figura 4, estão apresentados os valores de remoção dos micronutrientes em função da carga orgânica adicionada aos SACs.

O capim tifton-85 apresentou maior capacidade de remoção de $\mathrm{Cu}$, variando entre 5 e 30 vezes a remoção proporcionada pela taboa, provavelmente, pela maior quantidade de biomassa produzida. Quanto ao $\mathrm{Zn}$, somente no $\mathrm{SAC}_{2}$, cultivado com taboa e que recebeu a menor taxa de carga orgânica, foi obtida maior remoção que nos SACs cultivados com capim tifton-85. No entanto, não se verificou diferença estatística $(\mathrm{p}<0,05)$, quanto à remoção de micronutrientes, nem entre as espécies cultivadas nem mesmo entre os tratamentos aplicados.

Matos et al. (2009) verificaram que a taboa e o capim tifton-85 foram responsáveis pela remoção média de $0,22 \mathrm{~kg} \mathrm{ha}^{-1}$ e $0,20 \mathrm{~kg} \mathrm{ha}^{-1}$ do $\mathrm{Cu}$, e $1,88 \mathrm{~kg} \mathrm{ha}^{-1}$ e 5,29 $\mathrm{kg} \mathrm{ha}^{-1}$ do $\mathrm{Zn}$ adicionados aos SACs utilizados no tratamento da ARS, no intervalo de 120 dias entre os cortes realizados nos vegetais. As maiores taxas de remoção obtidas por Matos et al. (2009) podem ter sido proporcionadas pelo maior aporte desses micronutrientes aos sistemas, da ordem de três a dez vezes ao que foi aplicado neste trabalho. Exceção deve ser feita no que se refere à remoção de Cu pelo capim tifton-85, que neste trabalho foi superior.

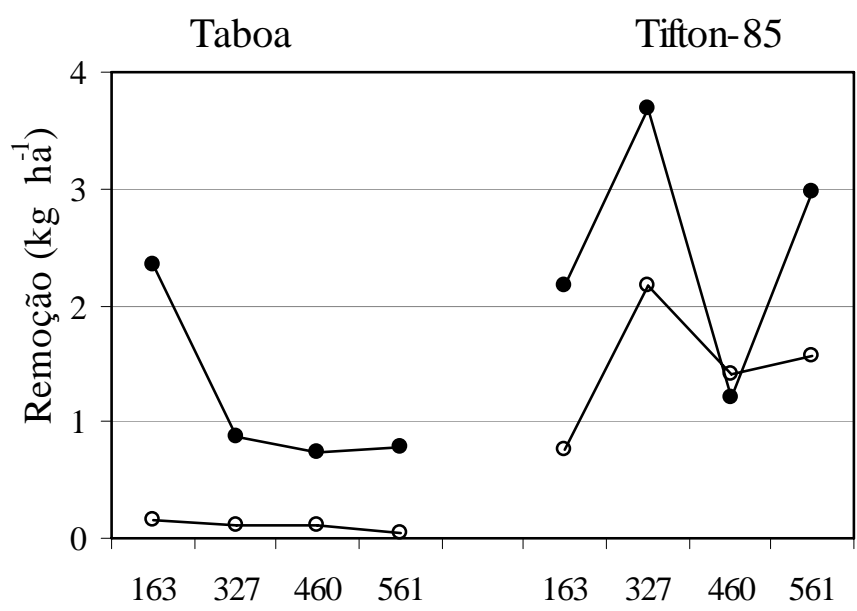

Cargas médias $\left(\mathrm{kg} \mathrm{ha}^{-1} \mathrm{~d}^{-1}\right.$ de DBO)

$$
\rightarrow \mathrm{Zn} \rightarrow \mathrm{Cu}
$$

Figura 4. Valores médios de remoção $\left(\mathrm{kg} \mathrm{ha}^{-1}\right)$ de cobre $(\mathrm{Cu})$ e zinco $(\mathrm{Zn})$, obtidos em dois cortes nas plantas, realizados em 120 dias de operação dos SACs, em função das cargas orgânicas aplicadas.

Queiroz et al. (2004) também aplicaram, durante quatro meses, maiores cargas de $\mathrm{Cu}$ (1,2 kg ha $\left.{ }^{-1} \mathrm{~d}^{-1}\right)$ e Zn (14,5 $\left.\mathrm{kg} \mathrm{ha}^{-1} \mathrm{~d}^{-1}\right)$ em capim tifton-85 por meio de fertirrigação com ARS 
e, ainda assim, as taxas de recuperação, por meio do corte da parte aérea do capim tifton-85, foram pequenas, sendo de $0,18 \mathrm{~kg} \mathrm{ha}^{-1}$ de $\mathrm{Cu}$ e $1,5 \mathrm{~kg} \mathrm{ha}^{-1}$ de $\mathrm{Zn}$, provavelmente pela complexação desses elementos pela matéria orgânica ou adsorção na matriz do solo. Silva (2008) não detectou concentrações de $\mathrm{Cu}$ e $\mathrm{Zn}$ na matéria seca do capim tifton-85 ao fertirrigá-lo com chorume de resíduos sólidos urbanos contendo concentrações de até 16,3 e 8,3 $\mathrm{mg} \mathrm{L}^{-1}$ de Cu e Zn.

Neste trabalho, verificou-se que o capim tifton-85 apresentou maior desenvolvimento vegetativo que a taboa, produzindo maior biomassa e, consequentemente, maior capacidade de remover macro e micronutrientes.

\section{CONCLUSÕES}

Com base nos resultados obtidos, pode-se concluir que a taboa não se adaptou às condições de exposição a altas cargas orgânicas e de nutrientes, não apresentando bom desempenho agronômico em termos de produtividade e extração de nutrientes, quando cultivada em SACs de tratamento de águas residuárias da suinocultura, nas taxas de carregamento orgânico avaliadas.

A produtividade média de matéria seca obtida em cada SAC em ambos os cortes da taboa variou entre 0,75 e 1,76 Mg ha ${ }^{-1}$ e do capim tifton-85 de 4,73 a 6,26 $\mathrm{Mg} \mathrm{ha}^{-1}$, após 60 dias de cultivo dessas espécies vegetais, sendo que as maiores produtividades ocorreram quando foi aplicada a carga orgânica média de $327 \mathrm{~kg} \mathrm{ha}^{-1} \mathrm{~d}^{-1}$ de DBO.

O capim tifton-85 foi a espécie vegetal que apresentou maior capacidade extratora de nutrientes, chegando a extrair entre 443 e 540, 86 e 99, 193 e 241, 0,77 e 2,17, e 1,21 e 3,68 kg ha-1 de NTK, P, K, Cu e Zn, respectivamente, durante 120 dias de cultivo das plantas nos SACs, enquanto a taboa apresentou maior capacidade de absorção de sódio.

\section{AGRADECIMENTOS}

Os autores agradecem à FAPEMIG pelo apoio financeiro concedido.

\section{REFERÊNCIAS}

AMERICAN PUBLIC HEALTH ASSOCIATION - APHA; AMERICAN WATER WORKS ASSOCIATION - AWWA; WATER ENVIRONMENT FEDERATION - WEF. Standard methods for the examination of water and wastewater. 21. ed. Washington:APHA/AWWA/WEF, 2005.

BERTIN, C.; YANG, X.; WESTON, L. A. The role of root exudates and allelochemicals in the rhizosphere. Plant Soil, v. 256, p. 67-83, 2003.

http://dx.doi.org/10.1023/A:1026290508166.

BRASIL, M. S.; MATOS, A. T.; SOARES, A. A. Plantio e desempenho fenológico da taboa (Typha sp.) utilizada no tratamento de esgoto doméstico em sistema alagado construído. Engenharia Sanitária e Ambiental, v. 12, n. 3, p. 266-272, 2007a.

BRASIL, M. S.; MATOS, A. T.; FIA, R.; LIMA, N. C. Desempenho agronômico de vegetais cultivados em sistemas alagados utilizados no tratamento de águas residuárias da suinocultura. Engenharia na Agricultura, v. 15, n. 3, p. 307-315, 2007 b. 
BRIX, H. Do macrophytes play a role in constructed treatment wetlands? Water Sci. Technol., v. 35, n. 5, p. 11-17, 1997. http://dx.doi.org/10.1016/S0273-1223(97)00047-4.

CHAGAS, R. C. Utilização de lírio amarelo (Hemerocallis flava) em sistemas alagados construídos para tratamento de esgoto doméstico. 2008. 66f. Dissertação (Mestrado em Engenharia Agrícola) - Universidade Federal de Viçosa, Viçosa, MG, 2008.

CLARKE, E.; BALDWIN, A. H. Responses of wetland plants to ammonia and water level. Ecological Engineering, v. 18, p. 257-264, 2002. http://dx.doi.org/10.1016/S0925-8574(01)00080-5.

Empresa Brasileira de Pesquisa Agropecuária - EMBRAPA. Manual de análises químicas de solos, plantas e fertilizantes. Brasília, EMBRAPA, 1999. 370p.

Food and Agriculture Organization of the United Nations - FAO. Handbook of utilization aquatic plants. Disponível em: <http://www.fao.org/DOCREP/003/X6862E/ X6862E03.htm>. Acesso em: 03 dez. 2007.

FIA, R.; MATOS, A. T.; FERREIRA, P. A.; TEODORO, P. E. P.; SCHUERY, F. C.; LUIZ, F. A. R. Desempenho agronômico da Typha sp. e Alternanthera philoxeroides Mart. utilizadas no tratamento de águas residuárias da lavagem e descascamento/despolpa dos frutos do cafeeiro em sistema alagado construído. Engenharia na Agricultura, v. 16, n. 4, p. 436-448, 2008.

FIA, R.; MATOS, A. T.; FIA, F. R. L.; MATOS, M. P.; LAMBERT, T. F.; NASCIMENTO, F. S. Desempenho de forrageiras em sistemas alagados de tratamento de águas residuárias do processamento do café. Revista Brasileira de Engenharia Agrícola e Ambiental, v. 14, n. 8, p. 842-847, 2010. http://dx.doi.org/10.1590/S1415-43662010000800008.

KADLEC, R. H.; TANNER, C. C.; HALLY, V. M.; GIBBS, M. M. Nitrogen spiraling in subsurface-flow constructed wetlands: implications for treatment response. Ecological Engineering, v. 25, p. 365-381, 2005. http://dx.doi.org/10.1016/j.ecoleng.2005.06.009.

KADLEC, R. H.; WALLACE, S. D. Treatment wetlands. 2. ed. Boca Raton: CRC Press, 2008. 1016p. http://dx.doi.org/10.1201/9781420012514.

LOURES, A. P. S.; SOARES, A. A.; MATOS, A. T.; CECON, P. R.; PEREIRA, O. G. Remoção de fósforo em sistema de tratamento de esgoto doméstico, por escoamento superficial. Revista Brasileira de Engenharia Agrícola e Ambiental, v. 10, n. 3, p. 706-714, 2006.

MATOS, A. T.; ABRAHÃO, S. S.; PEREIRA, O. G. Desempenho agronômico de capim tifton 85 (Cynodon spp.) cultivado em sistemas alagados construídos utilizados no tratamento de água residuária de laticínios. Revista Ambiente e Água, v. 3, n. 1, p. 43 53, 2008.

MATOS, A. T.; FREITAS, W. S.; LO MONACO, P. A. V. Capacidade extratora de diferentes espécies vegetais cultivadas em sistemas alagados utilizados no tratamento de águas residuárias da suinocultura. Revista Ambiente e Água, v. 4, n. 2, p. 31-45, 2009. 
MAZZOLA, M.; ROSTON, D. M.; VALENTIM, M. A. A. Uso de leitos cultivados de fluxo vertical por batelada no pós-tratamento de efluente de reator anaeróbio compartimentado. Revista Brasileira de Engenharia Agrícola e Ambiental, v. 9, n. 2, p. 276-283, 2005.

MEIRA, C. M. B. S.; CEBALlOS, B. S. O.; KÖNIG, A.; Oliveira, R. Performance of horizontal subsurface flow constructed wetlands vegetated with rice treating a sewage polluted surface water. In: INTERNATIONAL CONFERENCE ON WASTE STABILISATION PONDS, 6.; INTERNATIONAL CONFERENCE ON WETLAND SYSTEMS FOR WATER POLLUTION CONTROL, 9., 2004, Avignon. Proceedings... Avignon: IWA/Astee, 2004. 1 CD-ROM.

OLIVEIRA, M. A.; PEREIRA, O. G.; GARCIA, R.; OBEID, J. A.; CECON, P. R.; MORAES, S. A.; SILVEIRA, P. R. Rendimento e valor nutritivo do capim-tifton 85 (Cynodon spp.) em diferentes idades de rebrota. Revista Brasileira de Zootecnia, v. 29, n. 6, p. 1949-1960, 2000. Suplemento 1.

QUEIROZ, F. M. Avaliação de gramíneas forrageiras para tratamento de águas residuárias da suinocultura. 2000. 91f. Dissertação (Mestrado em Engenharia Agrícola) - Universidade Federal de Viçosa, Viçosa, MG, 2000.

QUEIROZ, F. M.; MATOS, A. T.; PEREIRA, O. G.; OLIVEIRA, R. A.; LEMOS, A. L. Características químicas do solo e absorção de nutrientes por gramíneas em rampas de tratamento de águas residuárias da suinocultura. Revista Engenharia na Agricultura, v. 12, n. 2, p. 77-90, 2004.

SASMAZ, A.; OBEK, E.; HASAR, H. The accumulation of heavy metals in Typha latifolia L. grown in a stream carrying secondary effluent. Ecological Engineering, v. 33, p. 278-284, 2008.

http://dx.doi.org/10.1016/j.ecoleng.2008.05.006.

SILVA, D. F. Efeito da aplicação de percolado de resíduos sólidos urbano em solo cultivado com capim-tifton 85. 2008. 148f. Tese (Doutorado em Engenharia Agrícola) - Universidade Federal de Viçosa, Viçosa, MG, 2008.

SOUSA, J. T.; VAN HAANDEL, A. C.; COSENTINO, P. R. S.; GUIMARÃES, A. V. A. Pós-tratamento de efluente de reator UASB utilizando sistemas "wetlands" construídos. Revista Brasileira de Engenharia Agrícola e Ambiental, v. 4, n. 1, p. 87-91, 2000. 